\title{
Free convection from a point source of heat, and heat transfer from spheres at small Grashof numbers
}

\author{
V.N. Kurdyumov, A. Liñán
}

\section{Introduction}

The heat leaving hot particles in an unbounded fluid environment is confined, due to the buoyancy forces, to a heated region surrounding the particle and to a thermal plume above. Under quasi-steady conditions, the downwards extent or characteristic size, $l_{\mathrm{h}}$, of the heated region - and hence the rate $Q$ of loss of heat of the particle to the surrounding fluid-is determined by the balance of the transport of heat by conduction and by the convective flow. This flow is due only to the buoyancy forces in the cases considered in this paper when there is not an externally imposed forced flow.

The analysis of free convective flows and heat transfer from hot particles has received considerable attention; see, for example, the extended review of Gebhart et al. [1]. Most of the work refers to the cases, with large Grashof numbers, when the heat leaving the particle is confined to a thin boundary layer and to the plume above the particle. The heat transfer in this case is enhanced above the pure heat conduction value by a factor of the order of the ratio of the particle size to the thickness of the boundary layer. Merk and Prins [2] used an integral method to obtain the relation between the Nusselt and Grashof numbers in the large Grashof number limit. Acrivos [3] analysed the case of large values of the Prandtl number. Potter and Riley [4] studied the steady boundary layer flow over a heated sphere using a finite difference method; the calculation ends in a singularity when reaching the upper pole, from which the boundary layer erupts into the plume above the sphere. Additional detailed features of the singularity at the upper pole were revealed by Brown and Simpson [5].

In the opposite limiting case of small Grashof numbers the size $l_{\mathrm{h}}$ of the heated region is large compared with the body size and, then, the heat transfer is, in 


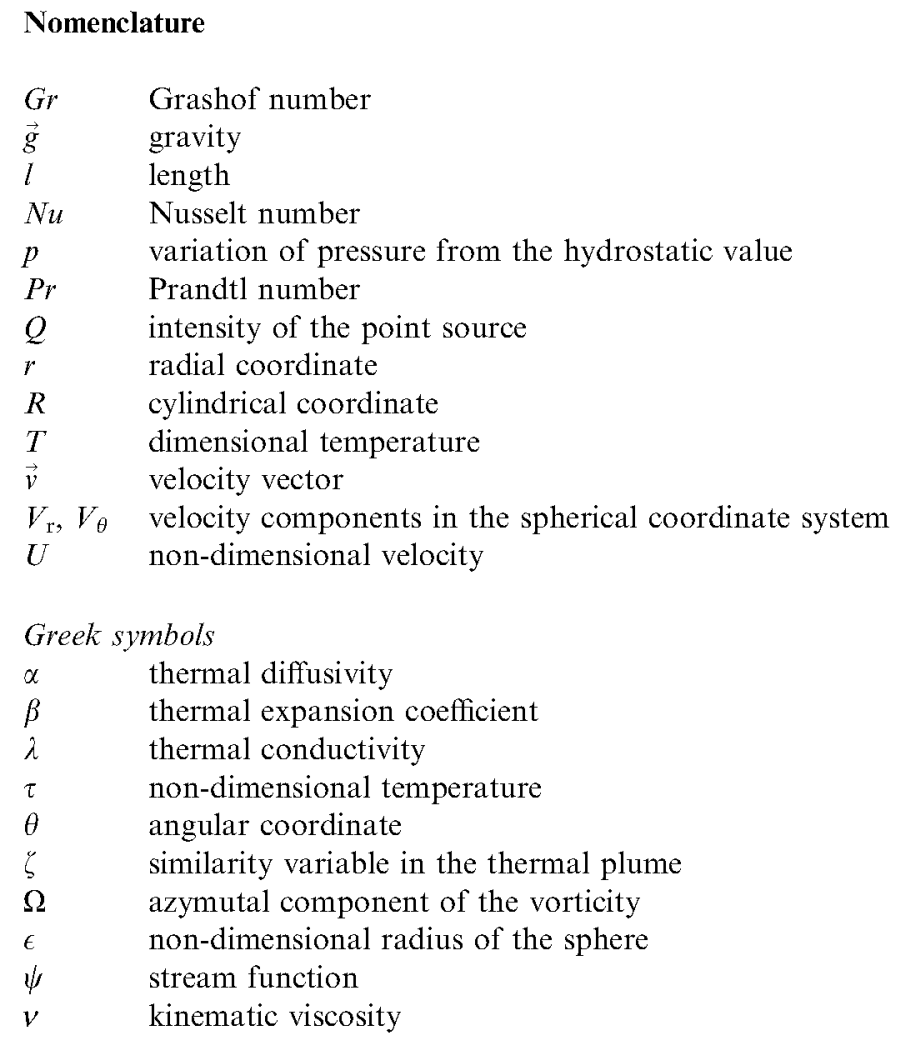

first approximation, determined by conduction for non-cylindrical bodies of finite size. For this reason, the cases of small Grashof numbers have received less attention in the literature.

The problem encountered in the description of the free convection flow at small Grashof numbers is a singular perturbation problem, as it was understood by Mahony [6], by Fendell [7], and very clearly by Hieber and Gebhart [8], who analysed the mixed convection around a heated sphere at small Reynolds and Grashof numbers. The flow field, and the correction due to convection of the heat flux from its heat conduction value, can only be obtained with the numerical solution of the complete equations or using the technique of matched asymptotic expansions, as in the analysis of the low Reynolds number past spheres of Kaplun and Lagerstrom [9]. This is what will be done in this paper, as we did in the analysis of the free convection flow due to thin heated wires, Liñan and Kurdyumov [10].

As pointed out by Hieber and Gebhart [8], in Sections 1.3 and 3 of their paper, there are two main regions in the free convection flow field around heated spheres. An inner region, scaled with the radius, $a$, of the sphere where the first terms of the expansion of the flow variables in powers of $G r^{1 / 2}$, the square root of the Grashof number, are given by solutions of the Stokes simplified form of the complete flow equations, without the terms due to the buoyancy forces and the convective transport of momentum and heat. The outer boundary conditions for these equations are obtained by matching with the solution of the complete equations describing the flow in an outer region, with a scale $l_{\mathrm{h}}$, of the order of $a G r^{-1 / 2}$, determined by the balance of heat conduction and the convective transport resulting from the flow induced by the buoyancy forces. The flow and temperature fields in this outer region are determined by the rate of heat loss $Q$ by the heated particle independently of its shape and size - the radius $a$ in the case of sphere. The heated particle appears in the low Grashof number limit as a pure point source of heat when we observe the flow with the outer scale, because the effect of the drag of the particle in the outer flow field can be neglected in this limit.

As we shall see below, the temperature variations $\left(T_{\mathrm{h}}-T_{\infty}\right)$ encountered in the outer region are smaller than the temperature difference $\left(T_{\mathrm{p}}-T_{\infty}\right)$ between the particle and the ambient fluid by a factor of the order $G r^{1 / 2}$. Typically $T_{\mathrm{h}}-T_{\infty} \ll T_{\infty}$, so that we can use the 
Boussinesq approximation of the conservation equations for the description of the outer solution corresponding to a point source of heat.

Then, we shall begin in Section 2, with a description of the numerical solution of the Boussinesq equations corresponding to the steady, laminar, free convection flow due to a point source of heat. When these equations are written in non-dimensional form using the appropriate scales only one parameter, namely the Prandtl number $P r$, is left in the equations and boundary conditions. The solution has singularities both for large and small values of the non-dimensional radial coordinate; and these must be described analytically with coordinate expansions to obtain the numerical solution. The analytical near source description involves constants, already identified by Hieber and Gebhart [8], that must be obtained, together with the numerical solution, in terms of the Prandtl number.

The far field includes a slender plume above the point source, driven by the buoyancy forces, and an outer irrotational flow, where $T=T_{\infty}$, due to the entrainment flow generated by the plume. The far field is the same for the free convection flow around heated bodies when the Grashof number is of order unity, determined again by $Q$, independently of the particle size and shape. Thus, this far field description will be used, in Section 3, in our numerical analysis of the free convection flow around heated spheres.

The equations describing the flow and temperature fields in the plume far above the particle can be simplified using approximations of the boundary layer type, and their asymptotic solution, as shown by Zeldovich [11], is self-similar both in the laminar and turbulent case, determined by the rate of heat loss $Q$ from the particle. The equations which describe the self-similar asymptotic structure of the plume were obtained by Yih [12], who showed that analytical solutions exist for the values 1 and 2 of the Prandtl number, and numerically solved by Fujii [13]. The asymptotic structure for $P r \gg 1$ of the plume above a point source has been analysed recently by Vázquez et al. [14]. Second-order effects in an axisymmetric plume in an unbounded fluid and a fluid bounded by a ground plate were considered by Thomas and Takhar [15], and by Riley and Drake [16] with an external co-flowing uniform stream for $P r=1$.

Geoola and Cornish [17] obtained the numerical solution of the full steady Navier-Stokes equations to calculate the natural convection heat transfer over a sphere for $P r=0.72$. However, the boundary condition, $\psi=0$, used for the stream function at large values of the radial coordinate does not correspond to the steady solution in an unbounded environment. When using this boundary condition an artificial recirculation zone appears, which may influence the heat transfer from the sphere. Calculations using the full steady
Navier-Stokes equations with similar, artificial, boundary conditions were also carried out by Jie and Gogos [18]. Transient flow calculations were carried out numerically for Grashof numbers up to $10^{5}$ by Geoola and Cornish [19]. Riley [20] obtained transient numerical solutions for $\operatorname{Pr}=0.72$ and $\operatorname{Pr}=7$ in the range $10^{2}$ $\leq G r \leq 10^{4}$, and compared the heat transfer results with those obtained from the boundary layer theory in the high Grashof number limit.

The boundary conditions appropriate for the flow around the sphere in an unbounded fluid environment must be based on the asymptotic description of the far flow field, as indicated above and used below, in Section 3, in our numerical analysis of the laminar flow around the sphere. The problem is posed so as to find the surface temperature of the sphere that results in a given heat flux. The numerical calculations were carried out for $P r=0.72$ and $P r=7$ for small and moderate values of the Grashof number. The method of matched asymptotic expansions is used in Section 3.2 to calculate the correction of the Nusselt number due to free convection effects at Grashof numbers small compared with unity. This is done by using the matching conditions between the inner heat conduction dominated solution and the outer solution due to a point heat source.

The results of the asymptotic description for large and small values of the Grashof number have been used to propose a correlation of the Nusselt number and Grashof numbers, valid for all values of $G r$ as long as the boundary layer flow is laminar.

\section{Free convection from a point source of heat}

\subsection{Formulation}

Consider a point source of heat of intensity $Q$ in an unbounded fluid with the temperature $T_{\infty}$ at the infinity, and subject to the gravity field $\vec{g}$. The equations that describe the flow and temperature fields can be simplified to the Boussinesq form if the temperature rise, $T-T_{\infty}$, is small compared with $T_{\infty}$. The density $\rho$, kinematic viscosity $v$, the thermal diffusivity $\alpha$, and the thermal expansion coefficient $\beta$, equal to $1 / T_{\infty}$ for gases, are considered to be constant. Then, the velocity $\vec{v}$, temperature $T$, and variation of pressure $p^{\prime}$ from the hydrostatic value satisfy the equations

$\nabla \cdot \vec{v}=0$

$(\vec{v} \cdot \nabla) \vec{v}=-\nabla p^{\prime} / \rho-\vec{g} \beta\left(T-T_{\infty}\right)+v \Delta \vec{v}$,

$(\vec{v} \cdot \nabla) T=\alpha \Delta T$. 
Without an external forcing flow, the free convection flow due to a point source of heat will be axisymmetrical around the vertical line through the point source. If we use a system of spherical coordinates, the temperature and flow field will depend only on the radial coordinate $r$, measured from the point source, and the angle $\theta$, measured from the vertical direction, opposite to $\vec{g}$.

To account for the point source of heat we require

$\lim _{r \rightarrow 0} 2 \pi \lambda r^{2} \int_{0}^{\pi} \frac{\partial T}{\partial r} \sin \theta \mathrm{d} \theta=-Q$,

where $\lambda$ is the thermal conductivity. For the pure point source of heat there is no similar momentum source or sink at $r=0$; however, we shall find there a vertical non-zero velocity, $u_{0}$, induced by the buoyancy forces. For a point heat source in an unbounded environment, $T-T_{\infty}$ and the velocity $\vec{v}$ must tend to zero when $r \rightarrow$ $\infty$, outside a slender vertical plume where the peak velocity will tend to a constant.

In the free convection problem formulated above, the only parameters entering in the equations and boundary conditions are the Prandtl number, $\operatorname{Pr}=\boldsymbol{v} / \alpha$, and the dimensional parameters $g \beta, Q / \lambda$ and $\alpha$, which define the scales $l_{\mathrm{h}}, v_{\mathrm{h}}$ and $T_{\mathrm{h}}-T_{\infty}$

$$
\begin{gathered}
l_{\mathrm{h}}=\left(\alpha^{2} \lambda / Q \beta g\right)^{1 / 2}, \quad v_{\mathrm{h}}=(g \beta Q / \lambda)^{1 / 2}, \\
T_{\mathrm{h}}-T_{\infty}=\left(Q^{3} \beta g / \alpha^{2} \lambda^{3}\right)^{1 / 2}
\end{gathered}
$$

These are the characteristic values, respectively, of the length of the heated region around the source and the velocity and temperature rise above the ambient in this region. If we use these scales, or the similar scales proposed by Hieber and Gebhart [8], to write the equations and boundary conditions in non-dimensional form, we obtain

$$
V_{\mathrm{r}} \frac{\partial \tau}{\partial r}+\frac{V_{\theta}}{r} \frac{\partial \tau}{\partial \theta}=\frac{\partial^{2} \tau}{\partial r^{2}}+\frac{2}{r} \frac{\partial \tau}{\partial r}+\frac{1}{r^{2}} \frac{\partial^{2} \tau}{\partial \theta^{2}}+\frac{\operatorname{ctg} \theta}{r^{2}} \frac{\partial \tau}{\partial \theta},
$$

$$
\begin{gathered}
\frac{\partial}{\partial r}\left(V_{\mathrm{r}} \Omega\right)+\frac{1}{r} \frac{\partial}{\partial \theta}\left(V_{\theta} \Omega\right)=-\left(\frac{\partial \tau}{\partial \theta} \cos \theta+r \frac{\partial \tau}{\partial r} \sin \theta\right) \\
+\operatorname{Pr}\left(\frac{\partial^{2} \Omega}{\partial r^{2}}+\frac{1}{r^{2}} \frac{\partial^{2} \Omega}{\partial \theta^{2}}+\frac{\operatorname{ctg} \theta}{r^{2}} \frac{\partial \Omega}{\partial \theta}-\frac{\Omega}{r^{2} \sin ^{2} \theta}\right),
\end{gathered}
$$

$$
\frac{\partial^{2} \psi}{\partial r^{2}}+\frac{1}{r^{2}} \frac{\partial \psi}{\partial \theta^{2}}-\frac{\operatorname{ctg} \theta}{r^{2}} \frac{\partial \psi}{\partial \theta}=-\Omega \sin \theta,
$$

where $\tau=\left(T-T_{\infty}\right) /\left(T_{\mathrm{h}}-T_{\infty}\right)$,

$$
\begin{aligned}
& \Omega=r \frac{\partial V_{\theta}}{\partial r}+V_{\theta}-\frac{\partial V_{\mathrm{r}}}{\partial \theta}, \quad V_{\mathrm{r}}=\frac{1}{r^{2} \sin \theta} \frac{\partial \psi}{\partial \theta}, \\
& V_{\theta}=-\frac{1}{r \sin \theta} \frac{\partial \psi}{\partial r},
\end{aligned}
$$

$\psi$ is the stream function, and $\Omega / r$ is the azymutal component of the vorticity. Eqs. (6)-(8) are to be solved for $r>0$ with the boundary conditions

$\tau \rightarrow 0, \quad V_{\mathrm{r}} \quad$ and $\quad V_{\theta} \rightarrow 0 \quad$ at $\quad r \rightarrow \infty \quad(\theta \neq 0)$,

and the requirement that

$\lim _{r \rightarrow 0}\left(4 \pi r^{2} \frac{\partial \tau}{\partial r}\right)=-1$

associated with the concentrated point heat source at $r=0$. Only one parameter, namely the Prandtl number, is left in the formulation (6)-(11).

\subsection{Asymptotic steady solutions for small and large $r$}

In the vicinity of the point source the temperature, stream function and vorticity can be described by the small $r$ expansions

$\tau=\frac{1}{4 \pi r}+A_{0}+\frac{U_{0}}{8 \pi} \cos \theta+O(r)$,

$\psi=\left(\frac{1}{2} U_{0} r^{2}-\frac{r^{3}}{32 \pi P r}\right) \sin ^{2} \theta+O\left(r^{4}\right)$,

$\Omega=\frac{r}{8 \pi P r} \sin \theta+O\left(r^{2}\right)$,

which include solutions of the Stokes equations not having a more singular behaviour than the solution, 1/ $4 \pi r$, required by the point source of heat. The constants appearing in the coordinate expansions (12) (14) must be determined as part of the numerical solution of the general point source problem. The constant $A_{0}$ determines the temperature level near the source, the constant $U_{0}$ measures the vertical velocity of the fluid at the point source.

In the numerical solution we shall not use directly (12)-(14) near the point source of heat, but use instead the following weak form of the boundary conditions at $r=r_{\min } \ll 1$

$r^{2} \frac{\partial \tau}{\partial r}+\frac{1}{4 \pi}=r \frac{\partial \psi}{\partial r}-2 \psi=\Omega-\frac{r}{8 \pi P r} \sin \theta=0$,

which are based on (13) and (14) but do not involve unknown constants.

The stationary far field associated with a point source of heat, or any three-dimensional body, in an unbounded stagnant environment includes a slender 


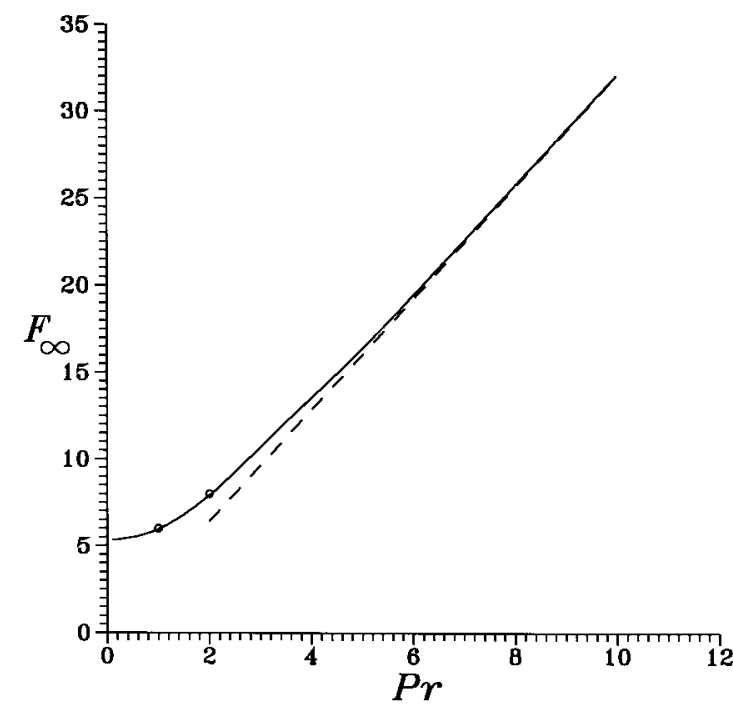

Fig. 1. Calculated values of $F_{\infty}$ as a function of the Prandtl number. Circles - the values, 6 and 8 , given by Yih [12] for $\operatorname{Pr}=1$ and 2. Dashed line - asymptotic behaviour, $F_{\infty}=3.2$ $\operatorname{Pr}$, obtained for $\operatorname{Pr} \rightarrow \infty$.

plume above the source, where the flow and temperature can be described in first approximation, for $r \gg 1$ and $\theta \sim \mathrm{r}^{-1 / 2}$, by

$\psi=r F(\zeta), \quad \tau=r^{-1} G(\zeta)$,

where the similarity variable, $\zeta=r^{1 / 2} \theta$, is of order unity in the plume. The well known equations determining the thermal plume are

$\zeta \frac{\mathrm{d} G}{\mathrm{~d} \zeta}=-F G$,

$\operatorname{Pr} \frac{1}{\zeta} \frac{\mathrm{d}}{\mathrm{d} \zeta}\left[\zeta \frac{\mathrm{d}}{\mathrm{d} \zeta}\left(\frac{1}{\zeta} \frac{\mathrm{d} F}{\mathrm{~d} \zeta}\right)\right]+\frac{F}{\zeta} \frac{\mathrm{d}}{\mathrm{d} \zeta}\left(\frac{1}{\zeta} \frac{\mathrm{d} F}{\mathrm{~d} \zeta}\right)+G=0$

to be solved, for $\zeta>0$, with the boundary conditions

$$
\begin{gathered}
F=\frac{\mathrm{d}}{\mathrm{d} \zeta}\left(\frac{1}{\zeta} \frac{\mathrm{d} F}{\mathrm{~d} \zeta}\right)=0 \quad \text { at } \zeta=0 ; \quad \frac{1}{\zeta} \frac{\mathrm{d} F}{\mathrm{~d} \zeta}=0 \quad \text { at } \\
\zeta \rightarrow \infty
\end{gathered}
$$

In addition, the functions $F$ and $G$ must satisfy the integral condition

$2 \pi \int_{0}^{\infty}(\mathrm{d} F / \mathrm{d} \zeta) G \mathrm{~d} \zeta=1$

stating that the heat transported upwards by the flow in the plume equals the heat leaving the source.

The numerical solution of the Eqs. (17)-(20) determines the value $F(\zeta)_{\zeta \rightarrow \infty}=F_{\infty}$ shown in Fig. 1 as a function of the Prandtl number. The closed-form solutions given by Yih [12] lead, for $P r=1$ and 2, to the values 6 and 8 of $F_{\infty}$, shown in Fig. 1 with circles. Shown in this figure with a dashed line is the asymptotic behaviour, $F_{\infty}=3.2 P r$, obtained for $\operatorname{Pr} \rightarrow \infty$. The values of the centre-line velocity, $U_{\mathrm{a}}=\left.\zeta^{-1}(\mathrm{~d} F / \mathrm{d} \zeta)\right|_{\zeta=0}$, are also shown in Fig. 5 with a dashed line.

For large $r$, outside the thermal plume, $T=T_{\infty}$, and the flow field is described by (8) with $\Omega=0$. As shown by Schneider [21], the appropriate potential flow solution for the stream function is

$\psi=\frac{F_{\infty}}{2} r(1+\cos \theta)$

corresponding to a semi-infinite volumetric line sink in an unbounded environment, of constant strength, $2 \pi F_{\infty}$, above the point heat source. It is interesting to observe that the radial velocity, $V_{\mathrm{r}}=-F_{\infty} / 2 r$, in the irrotational region is independent of $\theta$.

At large values of $r, r=r_{\max } \gg 1$, the stream function can be represented by a composite expression, based on the solutions for the plume and the irrotational regions, as

$\psi_{\mathrm{c}}=r_{\max }\left\{F\left(r_{\max }^{1 / 2} \theta\right)+\frac{1}{2} F_{\infty}(-1+\cos \theta)\right\}$

The corresponding expressions for the temperature and vorticity are given by (16), because in the inviscid region both are equal to zero.

Notice that according to (21), $\psi$ grows linearly with $r$ when $r \rightarrow \infty$. If the condition $\psi=0$ is posed at a finite although large value of $r$, as it has been done often in numerical descriptions of steady free convection flows in unbounded fluids, an artificial recirculation torus is generated, with a position depending on the size of the computational domain. The boundary condition $\psi=0$ at $r \rightarrow \infty$ can be used only during the transient period following the time of introduction of the source.

Using the expansion (22), the outer boundary, $r=r_{\max }$, is divided into inflow and outflow zones, with negative and positive values of the radial velocity. The dividing line, $\theta=\theta_{*}\left(r_{\max }\right)$, is determined by the extremum of $\psi\left(r_{\max }, \theta\right)$.

The following boundary conditions were adopted at $r=r_{\max }$ :

$$
\begin{gathered}
\psi-\psi_{\mathrm{c}}=\Omega=\tau=0, \quad \theta>\theta_{*} ; \\
\frac{\partial^{2} \psi}{\partial r^{2}}=\frac{\partial \Omega}{\partial r}=\frac{\partial \tau}{\partial r}=0, \quad \theta<\theta_{*}
\end{gathered}
$$



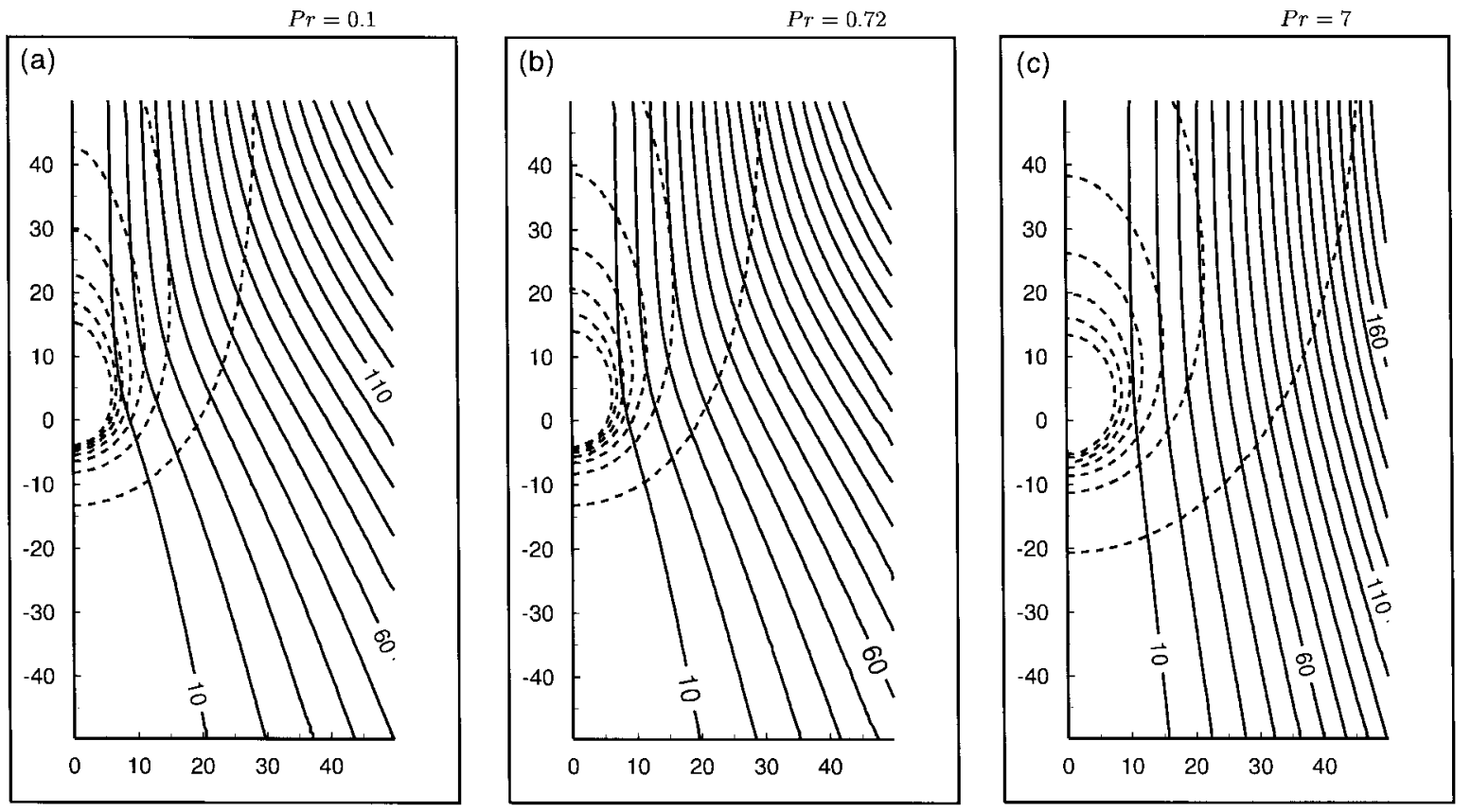

Fig. 2. Computed streamlines around the point source of heat (solid lines, $\psi$ at intervals of 10 ) and isotherms (dashed lines, $\tau$ at intervals 0.001 and $\tau_{\text {min }}=0.001$ ). (a) $\operatorname{Pr}=0.1$, (b) $\operatorname{Pr}=0.72$, (c) $\operatorname{Pr}=7$.

\subsection{Numerical solution of the steady point source problem}

The vorticity transport and energy equations, written in terms of $\ln r$ and $\theta$, were solved numerically, using second order, three points, approximations for the first and second derivatives. To obtain stationary distri-

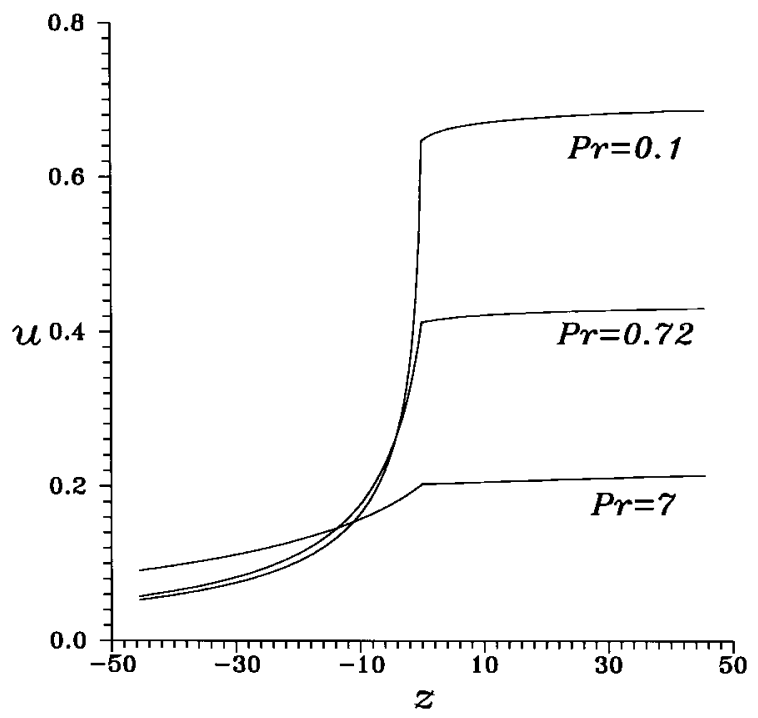

Fig. 3. Computed vertical velocity in the centre-line of the plume for various $P$ r. butions a pseudo unsteady form of the governing equations was used. The equation for the stream function was solved iteratively introducing an artificial time. Calculations were carried out using $101 \times 101$ and $151 \times 151$ points to test the grid dependence. It was admitted that the stationary distribution had been reached when $\max _{i, j}\left|f_{i, j}-\hat{f}_{i, j}\right|<10^{-10}$, where $f$ and $\hat{f}$ are the values of the current and previous time level, respectively. The results of the calculations shows that

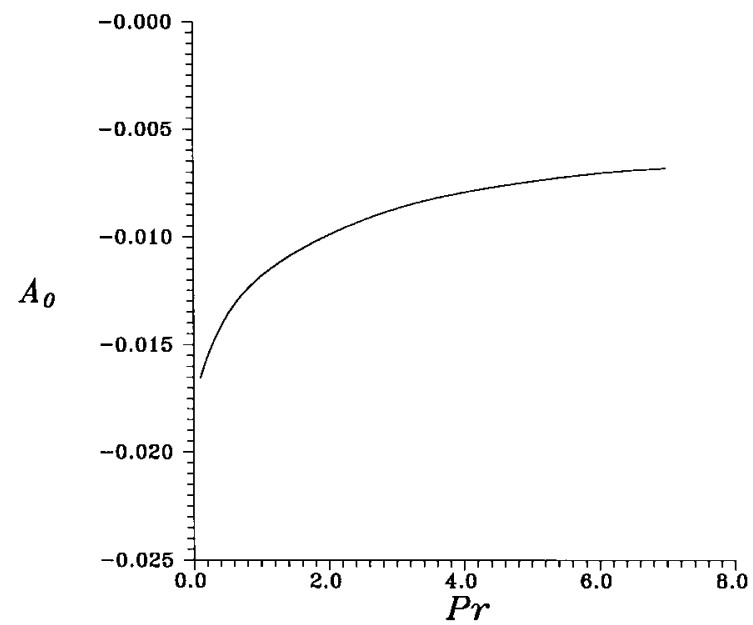

Fig. 4. Calculated values of the non-dimensional temperature level as a function of the Prandtl number. 


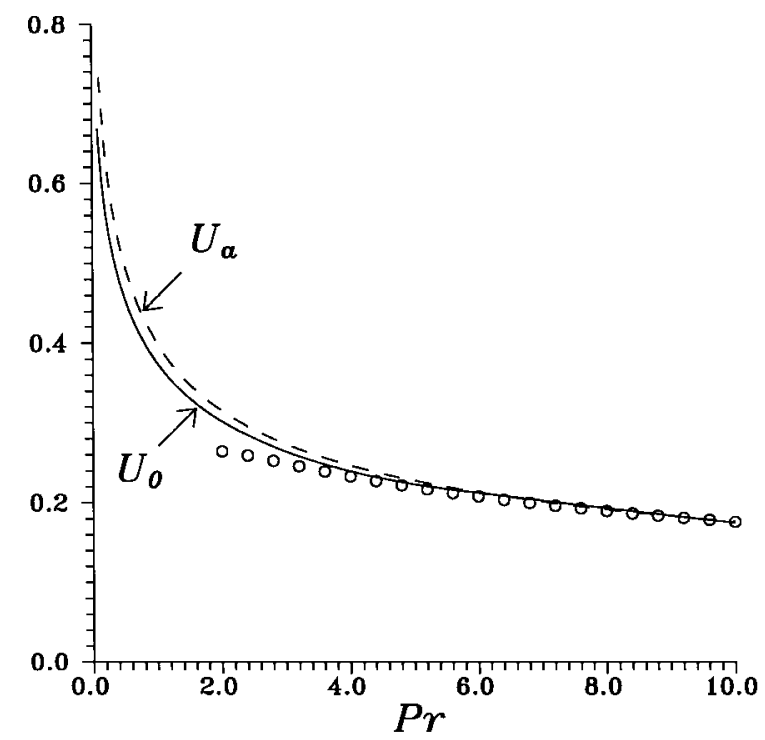

Fig. 5. Computed values of the point source velocity $U_{0}$ (solid line) and the far field velocity $U_{a}$ (dashed line) in the centreline of the plume. Circles - the fitting formula for this velocity proposed for large Prandtl numbers by Vázquez et al. [14].

the dependence of $A_{0}$ and $U_{0}$ on $r_{\min }$ and $r_{\max }$ disappears when $r_{\max }>100$ and $r_{\min }<10^{-3}$ in the range, between 0.1 and 10, of the Prandtl numbers considered in the calculations.

Typical results from the computations for streamlines and isotherms are shown in Fig. 2(a)-(c) with solid and dashed lines, respectively. These figures illustrate the changes in flow structure with increasing values of the Prandtl number. The velocity distribution along the vertical axis is shown in Fig. 3 for various values of $P r$. The values of $A_{0}$ and $U_{0}$, depending on the Prandtl number, are plotted in Figs. 4 and 5 with solid lines. The asymptotic value $U_{\mathrm{a}}$ of the vertical velocity in the plume is also shown in Fig. 5 with dashed lines. Shown also in Fig. 5 with circles is the asymptotic formula $U_{\mathrm{a}}=(0.38+0.67 \sqrt{\ln P r}) / \sqrt{2 \pi P r}$, proposed by Vázquez et al. [14] for $P r \gg 1$ for the velocity in the centre line of the plume, measured with our scale $v_{\mathrm{h}}$. One can see that the difference between $U_{0}$ and $U_{\mathrm{a}}$ tends to zero when $\operatorname{Pr} \rightarrow \infty$, and that the value of the velocity above the point source is nearly constant along the vertical axis.

\section{Steady free convection heat transfer from spheres at small Grashof numbers}

\subsection{Formulation and numerical description for $\mathrm{Gr} \sim 1$}

In this section we consider the steady laminar free convection around a sphere, posing the problem of

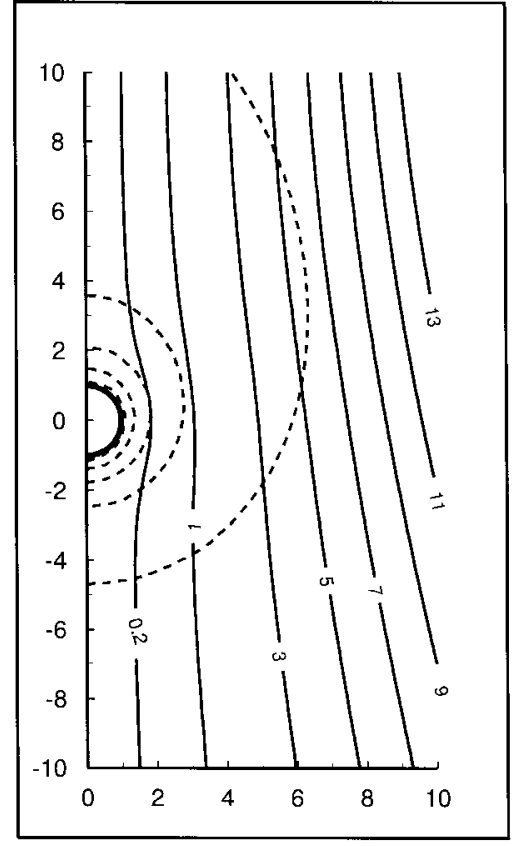

Fig. 6. Computed streamlines around the sphere for $\epsilon=1$ and $\operatorname{Pr}=0.72$ (solid lines) and normalized isotherms, $\tilde{\tau}=\tau / \tau_{\mathrm{p}}$ (dashed lines, $\tilde{\tau}$ at intervals of $0.2, \tilde{\tau}_{\min }=0.1$ ).

finding the uniform value of the surface temperature leading to a given rate $Q$ of heat loss. The description will be carried out using the non-dimensional Boussinesq Eqs. (6)-(8), based on the scales $l_{\mathrm{h}}, v_{\mathrm{h}}$ and $T_{\mathrm{h}}-T_{\infty}$, defined in (5), to be solved numerically with the following boundary conditions at the sphere surface, $r=\epsilon=a / l_{\mathrm{h}}$,

$\vec{v}=0,\left.\quad 2 \pi \int_{0}^{\pi} r^{2} \frac{\partial \tau}{\partial r}\right|_{r=\epsilon} \sin \theta \mathrm{d} \theta=-1, \quad \tau=\tau_{\mathrm{p}}$.

The value of the non-dimensional surface temperature, $\tau_{\mathrm{p}}=\left(T_{\mathrm{p}}-T_{\infty}\right) /\left(T_{\mathrm{h}}-T_{\infty}\right)$, assumed to be uniform, has to be determined as part of the solution as a function of $\epsilon$ and $P r$. Then, we obtain in parametric form the relation between the Nusselt and Grashof numbers, defined by

$$
\begin{aligned}
& N u=\frac{Q}{2 \pi \lambda a\left(T_{\mathrm{p}}-T_{\infty}\right)}=\frac{1}{2 \pi \tau_{\mathrm{p}} \epsilon}, \\
& G r=\frac{\beta g a^{3}\left(T_{\mathrm{p}}-T_{\infty}\right)}{v^{2}}
\end{aligned}
$$

The parametric representation for a given value of the Prandtl number is based on the non-dimensional radius of the sphere

$\epsilon=a / l_{\mathrm{h}}=\left(2 \pi N u G r P^{2}\right)^{1 / 2}$. 


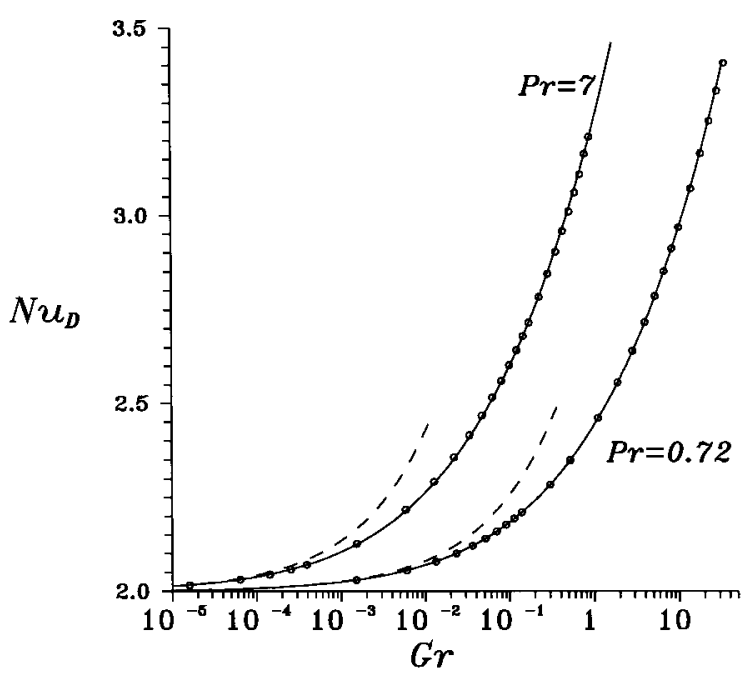

Fig. 7. Average Nusselt number as a function of $G r$ for $\operatorname{Pr}=0.72$ and 7 : circles - numerical results; solid lines-correlation formula (32); dashed lines-asymptotic behaviour (30) for small $G r$.

In the limit $G r \rightarrow 0$, it appears that the free convection effects on heat transfer become negligible, $\tau_{\mathrm{p}} \rightarrow 1 /(4 \pi \epsilon)$ and $N u=2$, in first approximation; while the flow velocities are of order $\epsilon$ when measured with $\alpha / a$.

Calculations were carried out for a wide range of $G r$ down to $10^{-4}$. Fig. 6 presents the computed isotherms and isolines of the stream function obtained for $\epsilon=1$ and $P r=0.72$. The resulting values of the mean Nusselt number as a function of the Grashof number are shown in Fig. 7 with circles for $P r=0.72$ and $\operatorname{Pr}=7$.

\subsection{Steady heat transfer at $\mathrm{Gr} \ll 1$ and correlation formula}

For values of $G r \ll 1$, and $P r=O(1), \epsilon=a / l_{\mathrm{h}} \ll 1$; then, two regions are found in the flow and temperature fields, with disparate scales $l_{\mathrm{h}}$ and $a$. In the outer region, with the scale $l_{\mathrm{h}}$, the flow can be approximated, in first approximation, by the flow induced by a point source with the strength $Q$. In the inner region near the sphere, scaled with its radius $a$, the flow can be described, in first approximation, by the stream function

$\psi=\frac{1}{2} U_{0}\left(r^{2}-\frac{3}{2} \epsilon r+\frac{1}{2} \frac{\epsilon^{3}}{r}\right) \sin ^{2} \theta$

the solution of the Stokes equations associated with the free convection flow velocity $U_{0}$ induced at the point source. The corresponding solution of the temperature equation, for $r \sim \epsilon, \epsilon \ll 1$, is of the form

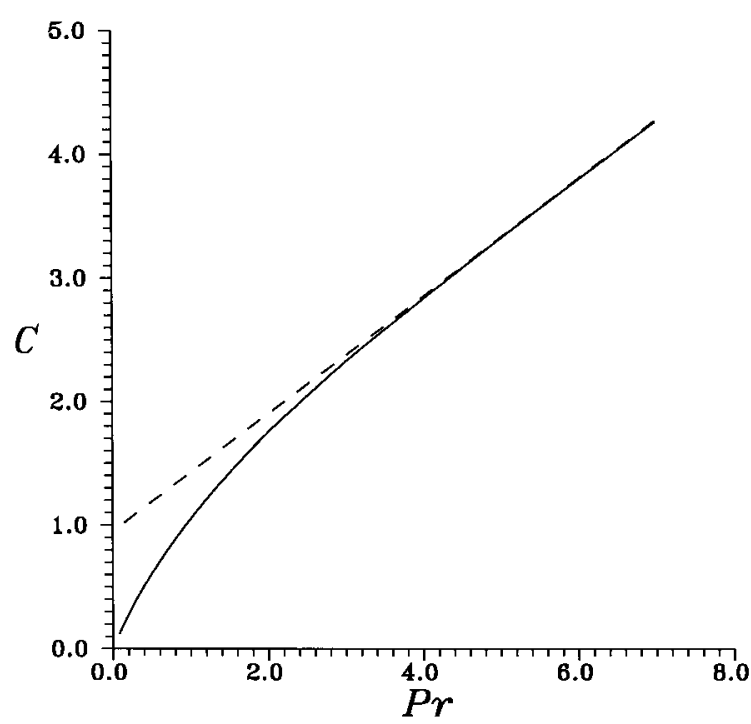

Fig. 8. Calculated values of $C(P r)$ as a function the Prandtl number (solid line); dashed line-correlation formula $C=0.474 \operatorname{Pr}+0.95$.

$$
\begin{aligned}
\tau & =\frac{1}{4 \pi r}+B_{0}+\frac{U_{0}}{8 \pi}\left(1-\frac{3}{2} \frac{\epsilon}{r}+\frac{3}{4} \frac{\epsilon^{2}}{r^{2}}-\frac{1}{4} \frac{\epsilon^{3}}{r^{3}}\right) \cos \theta \\
& +\ldots
\end{aligned}
$$

The requirement that (28) and (12) coincide in the intermediate region $\epsilon \ll r \ll 1$ leads to $B_{0}=A_{0}$. Thus, the following relation is obtained:

$\tau_{\mathrm{p}}=1 /(4 \pi \epsilon)+A_{0}$

The first term $1 / 4 \pi \epsilon$ in (29) corresponds to pure heat conduction, determining the surface temperature in terms of the rate of heat loss by the sphere. The negative correction $A_{0}$ corresponds to an apparent shift $\left(T_{\mathrm{h}}-T_{\infty}\right) A_{0}$, due to free convection, of the temperature of the environment seen by the sphere. Using (29) with (25) and (26) the following is obtained:

$N u=\frac{2}{1+4 \pi A_{0} \epsilon}=2+C(P r) G r^{1 / 2}, \quad G r \rightarrow 0$

where $C(P r)=-16 \pi^{3 / 2} \operatorname{Pr} A_{0}(P r)$ is shown in Fig. 8 by a solid line. The small value of $A_{0}$ is compensated by the factor $16 \pi^{3 / 2}$, so the values of $C(P r)$ are of order unity when $\operatorname{Pr}=O(1)$. For $\operatorname{Pr} \rightarrow \infty$ the function $C(P r)$ can be correlated by $C=0.474 P r+0.95$, shown in Fig. 8 with a dashed line. This asymptotic relation (30) allows $N u$ as a function of $G r$ in the limit of small Grashof numbers to be calculated.

At large Grashof numbers the relation between $N u$ and $G r$ takes the well known asymptotic form

$N u /(P r G r)^{1 / 4}=B(P r)$ 


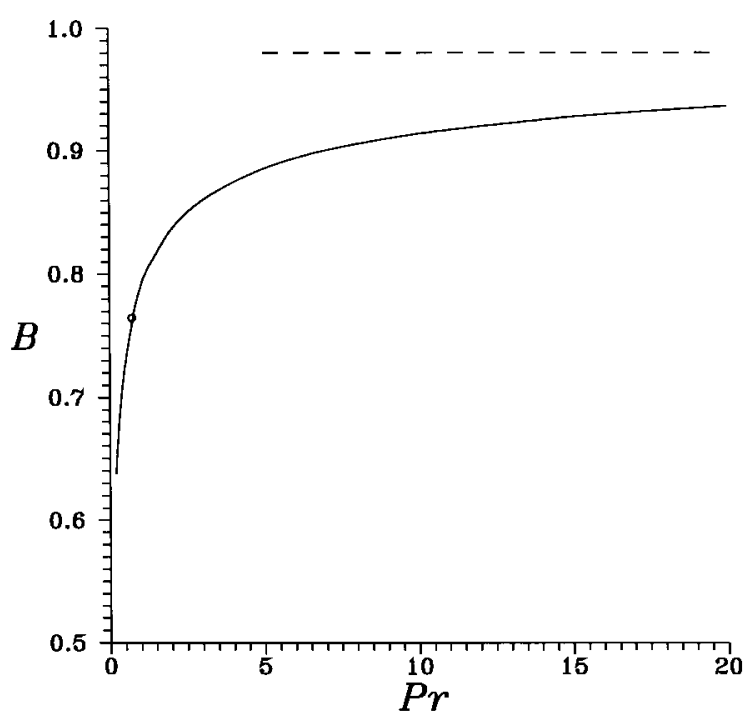

Fig. 9. Calculated values of the constant $B$ in the large Grashof number description (31); dashed line-asymptotic value, 0.98 , for $\operatorname{Pr} \rightarrow \infty$, obtained by Acrivos [3]; circle-the value 0.765 , calculated by Potter and Riley [4] for $P r=0.72$.

written in the form appropriate for the limit $\mathrm{Pr} \rightarrow \infty$, when $B \rightarrow 0.98$ as calculated by Acrivos [3], and shown in Fig. 9 with a dashed line. To calculate $B$ for $\operatorname{Pr}=0.72$, Potter and Riley [4] integrated numerically the corresponding boundary layer equations in $\theta$ from $\pi$ up to 0.12 , and extrapolated the local rate of heat transfer up to $\theta=0$. Our calculated values of $B$ as a function of the Prandtl number are shown in Fig. 9 where the boundary layer equations were integrated up to $\theta=0.14$, and then used the same method of the extrapolation of the local heat transfer rate up to $\theta=0$. The value, corresponding to $B=0.76$, calculated by Potter and Riley for $\operatorname{Pr}=0.72$, is shown with a circle.

To obtain a correlation formula for the Nusselt number, valid for the steady laminar free convection flow for all Grashof numbers, the following expression is used:

$N u=2+\frac{C(P r) G r^{1 / 2}}{1+s_{0} G r^{1 / 8}+s_{1} G r^{1 / 4}}$,

which coincides with [30] for $G r \rightarrow 0$ and with (31) for $G r \rightarrow \infty$ if $s_{1}=C(P r) / B(P r)$. The adjustable parameter $s_{0}$ is chosen as -0.32 and -0.52 for $\operatorname{Pr}=0.72$ and $P r=7$, respectively. The resulting Nusselt numbers, shown in Fig. 7 with solid lines, correlate the values obtained from our numerical calculations with errors lower than $1.5 \%$.

\section{Conclusions}

The main aim of the work presented in this paper is to provide the description of the steady laminar free convection flow and heat transfer from heated spheres at small Grashof numbers. As in the analysis of the free convection flow due to thin heated wires, see Liñán and Kurdyumov [10], the problem is posed as to find the uniform temperature, $T_{\mathrm{p}}$, of a sphere leading to a given rate $Q$ of heat loss to the fluid. The governing Boussinesq equations were written in non-dimensional form using the scales given by (5), and only the Prandtl number and the value of the non-dimensional radius of the sphere, $\epsilon=a / l_{\mathrm{h}}$, were left as parameters in the non-dimensional formulation, which can be used for the numerical description for all values of the Grashof number.

For values of $G r \ll 1$ the corresponding values of $\epsilon=a / l_{\mathrm{h}}$ are also small compared with unity, if $\operatorname{Pr} \sim 1$. Then, two regions in the flow field were found, with disparate scales $l_{\mathrm{h}}$ and $a$. In the outer region, with scale $l_{\mathrm{h}}$, the flow and temperature fields correspond, in first approximation, to those associated with a pure point source of heat at the centre of the sphere, with a strength corresponding to the rate of heat lost $Q$ by the sphere. The description of these fields is given in Section 2, based on the Boussinesq approximate form of the complete conservation equations, which involve the Prandtl number as the only parameter. As part of the solution the free convection velocity $U_{0}$ at the source was obtained and the parameter $A_{0}$ characterizing the temperature level near the source.

$U_{0}$ can be used to describe the flow field in the inner region around the sphere, where the equations can be simplified to the Stokes equations. The Stokes drag, $6 \pi \rho v^{2} v_{\mathrm{h}} U_{0}$, should be included as a point sink of momentum for a better description of the flow field in the outer region. However, this momentum sink is small, by a factor of the order $\epsilon P r$, when compared with the characteristic momentum flux $\rho v_{\mathrm{h}}^{2} l_{\mathrm{h}}^{2}=\rho \alpha^{2}$ in the outer region $r \sim l_{\mathrm{h}}$, and thus can be neglected for $\epsilon \ll 1$.

The value of $A_{0}$ was used, together with the matching conditions with the analytical solution for the inner region around the sphere, to calculate for $G r \ll 1$ the correction of the Nusselt number from its pure heat conduction value $N u=2$. This correction, given by the factor $\left(1+4 \pi A_{0} \epsilon\right)^{-1}$, corresponds to an apparent shift, $A_{0}\left(T_{\mathrm{h}}-T_{\infty}\right)$, of the temperature of the environment seen by the sphere. To obtain the corrections of the Nusselt number due to free convection at $G r \ll 1$ for particles of non-spherical shape, the pure heat conduction equation needs to be solved changing the far field temperature $T_{\infty}$ by $T_{\infty}+A_{0}\left(T_{\mathrm{h}}-T_{\infty}\right)$, because the far field at $G r \ll 1$ is determined by $Q$ independently of the particle shape. 
Numerically the complete Boussinesq equations were solved, for $P r=0.72$ and $P r=7$, for heated spheres with different values of the non-dimensional radius $\epsilon$; the Nusselt number was thus calculated for a wide range of Grashof numbers. Using the results of these calculations, together with the asymptotic expressions (30) and (31) for small and large values of the Grashof number, a correlation formula (32) applicable for all Grashof numbers is suggested.

The scales used in this analysis correspond to those applicable to values of the Prandtl number of order unity or small compared with unity. In these cases, the appropriate parameter that characterizes the size of the heated region surrounding the sphere is $G r P r^{2}$, and in the limit $P r \rightarrow 0$ the Nusselt number should be a function only of this parameter. In this limit, the viscous effects enter only in a boundary layer and a plume, which are very thin compared with the heated region and the thermal plume.

The cases with $P r \gg 1$ deserve particular attention and therefore these are considered in the Appendix. For values of the Rayleigh number, $R a=G r P r$, small compared with unity the size of the heated region around the sphere is much smaller than the size of the region where viscous transport of momentum is important; at small values of the Rayleigh number, this momentum is generated by the buoyancy forces acting on the slender plume above the sphere. The structures of the thermal plume and of the much larger surrounding region of viscous transport of momentum are determined by the heat released by the sphere, which appears as a point source of heat. The order of magnitude estimates, given in the Appendix, and the results of the numerical calculations, indicate that the ratio between the value, $u_{0}$, of the velocity in the point source, and the value, $u_{\mathrm{a}}$, of the centre-line velocity in the plume, tends to 0 when $P r \rightarrow \infty$. The approximate formula, proposed by V́azquez et al. [14], for the centre-line velocity in the plume can be used to estimate the effective Peclet number, $\sigma=O\left(R a^{1 / 2}\right)$, of the corresponding forced flow around the heated sphere. When the Rayleigh number grows to values of order unity the Nusselt number increases significantly above 2 , the heat conduction value, because the size of the heated region surrounding the sphere is of order $a$. In this paper this regime is not analysed, $R a \sim 1$, of transition to the boundary layer regime analysed, for $P r \gg 1$ and $R a \gg 1$, by Acrivos [3].

Although it may be expected that $\left(T_{\mathrm{h}}-T_{\infty}\right) / T_{\infty}$ is small compared with unity for $G r \ll 1$, and, then, the Boussinesq approximation will be justified in the outer region, when $\left(T_{\mathrm{p}}-T_{\infty}\right) / T_{\infty}$ is of order unity, this needs to be accounted, and this is easy to do, for the variation of the heat conduction with temperature in the heat conduction dominated inner region.

\section{Acknowledgements}

This research has been supported by Spanish DCICYT, under Contract No. PB94-0040, and by INTA, under Contract No. 4070-0036/1996.

\section{Appendix A}

Free convection from a sphere at large Prandt numbers

For values of $P r \gg 1$, the size $l_{\mathrm{h}}^{\prime}$ of the heated region around sphere, or heat source, will be small compared with the much larger size $l_{\mathrm{v}}$ of the region where viscous transport of momentum is important. We shall begin by giving estimates of these lengths for the point heat source, and we shall indicate, afterwards, how the flow and temperature fields around a sphere depend on the ratios $a / l_{\mathrm{h}}^{\prime}$ and $a / l_{\mathrm{v}}$.

In free convection flows at $P r \gg 1$, as it was the case for the line source of heat analysed by Liñan and Kurdyumov [10], the heated region around the source is continued above by a thermal plume of thickness $\delta_{\mathrm{T}} \ll l_{\mathrm{v}}$. This plume and the viscous region around the sphere are continued above by a slender plume where the flow, at distances $z \gg l_{\mathrm{v}}$, is self-similar as described in Section 2.2, and for large $\operatorname{Pr}$ by Vázquez et al. [14].

The buoyancy forces in the thermal plume are balanced by viscous stresses that extend outwards to the viscous region, driving there the flow with velocities of order $u_{\mathrm{v}}$, such that

$u_{\mathrm{v}} l_{\mathrm{v}} / v=1$

The equations describing the flow in the thermal plume, written in cylindrical coordinates $(z, R)$, can be simplified to the form

$\frac{\partial R u}{\partial z}+\frac{\partial R v}{\partial R}=0$

$0=g \beta\left(T-T_{\infty}\right)+v \frac{1}{R} \frac{\partial}{\partial R}\left(R \frac{\partial u}{\partial R}\right)$

$u \frac{\partial T}{\partial z}+v \frac{\partial T}{\partial R}=\frac{v}{P r} \frac{1}{R} \frac{\partial}{\partial R}\left(R \frac{\partial T}{\partial R}\right)$

where, because $\delta_{\mathrm{T}} / l_{\mathrm{v}} \ll 1$, the boundary layer approximation is justified and the convective terms in the momentum equation can be neglected.

From the energy equation the following is obtained

$\int_{0}^{\infty} \rho c_{\mathrm{p}} u\left(T-T_{\infty}\right) 2 \pi R \mathrm{~d} R=Q$,

while the momentum equation can be integrated across 
the thermal plume to yield

$$
\left(R \frac{\partial u}{\partial R}\right)_{\delta_{\varepsilon}}=-\int_{0}^{\infty}(g \beta / v)\left(T-T_{\infty}\right) R \mathrm{~d} R
$$

determining the viscous stresses at the outer edge $\delta_{\mathrm{e}}$ of the thermal plume.

If it is anticipated that the variation of $u$ across the thermal plume is of the order $u_{\mathrm{v}}$, moderately small compared with the centre-line velocity $u_{\mathrm{c}}$, so that $u$ can be approximated by $u_{\mathrm{c}}$ in (A5), then (A6) takes the form

$$
\left(R \frac{\partial u}{\partial R}\right)_{\delta_{\mathrm{e}}}=\frac{g \beta}{v} \frac{Q}{2 \pi \rho c_{\mathrm{p}} u_{\mathrm{c}}}
$$

This equation is applicable at radial distances such that $\delta_{\mathrm{T}} \ll R \ll l_{\mathrm{v}}$, where

$u-u_{\mathrm{c}}=-\left(g \beta Q / 2 \pi \rho c_{\mathrm{p}} v u_{\mathrm{c}}\right)(\ln R+c)$.

The matching conditions with the solution for the inner thermal plume, where $u=u_{\mathrm{c}}$ in first approximation, determines $c=-\ln \delta_{\mathrm{T}}$. By matching the solution for the outer viscous region, at $R \sim l_{\mathrm{v}}$ where $u \sim$ $u_{\mathrm{v}}$, it follows that

$g \beta Q / 2 \pi \rho c_{\mathrm{p}} v u_{\mathrm{c}}=u_{\mathrm{v}}$

and

$u_{\mathrm{v}} \ln \left(l_{\mathrm{v}} / \delta_{\mathrm{T}}\right)=u_{\mathrm{c}}$

An additional relation between $\delta_{\mathrm{T}}, l_{\mathrm{v}}$ and $u_{\mathrm{c}}$ comes from the required balance in the energy equation of convection and radial conduction of heat, so that

$\delta_{\mathrm{T}}=\sqrt{\alpha l_{\mathrm{v}} / u_{\mathrm{c}}}$

The relations (A1), (A10) and (A11) determine the scale ratios

$l_{\mathrm{v}} / \delta_{\mathrm{T}}=\sqrt{P r u_{\mathrm{c}} / u_{\mathrm{v}}} \quad u_{\mathrm{c}} / u_{\mathrm{v}}=\ln \sqrt{P r u_{\mathrm{c}} / u_{\mathrm{v}}}$

Notice that (A12) gives directly $u_{\mathrm{c}} / u_{\mathrm{v}}$ in terms of $P r$, and when used with (A9) the following is obtained

$$
\begin{aligned}
u_{\mathrm{c}} & =\left\{(g \beta Q / 2 \pi \lambda P r) \ln \sqrt{\operatorname{Pr}^{r} u_{\mathrm{c}} / u_{\mathrm{v}}}\right\}^{1 / 2} \\
& =\left(v_{\mathrm{h}} / \sqrt{2 \pi P r}\right)\left(\ln \sqrt{\operatorname{Pr} u_{\mathrm{c}} / u_{\mathrm{v}}}\right\}^{1 / 2}
\end{aligned}
$$

to calculate the characteristic vertical velocity in the plume. This result of the order of magnitude analysis is to be compared with the correlation proposed by Vázquez et al. [14] of their numerical results for the peak velocity, $u_{\mathrm{a}}$, in the self-similar plume; the factor $\left\{\ln \sqrt{P r u_{\mathrm{c}} / u_{\mathrm{v}}}\right\}^{1 / 2}$ is replaced by the factor $\{0.37+$ $0.67 \sqrt{\ln P r}$ in the correlation.
In the thermal plume, the vertical and transverse variations of the velocity from $u_{\mathrm{c}}$ are moderately small, of order $u_{\mathrm{v}}$, so that the velocity $u_{0}$ induced at the source is $u_{0}=u_{\mathrm{c}}$, given by (A12) and (A13).

This estimate can be used to calculate the size $l_{\mathrm{h}}^{\prime}$ of the heated region around the heat source, given by

$u_{\mathrm{c}} l_{\mathrm{h}}^{\prime} / \alpha=1$,

so that $l_{\mathrm{h}}^{\prime} / l_{\mathrm{v}}=u_{\mathrm{v}} / u_{\mathrm{c}} P r$, which is equal to $1 / \operatorname{Pr}$ aside from the moderately small factor $u_{\mathrm{v}} / u_{\mathrm{c}}$.

The order of magnitude estimates given above, and the results of the numerical calculations, show that the ratios of $u_{0}$ and $u_{\mathrm{a}}$ to $u_{\mathrm{c}}$ tend to 0 when $\operatorname{Pr} \rightarrow \infty$, so that the assumption $u_{0}=u_{\mathrm{a}}$, used by Fendell [7] in his analysis of the free convection heat transfer from spheres, is justified for large Prandtl numbers, and it can be written

$u_{0} \simeq u_{\mathrm{a}} \simeq u_{\mathrm{c}} \simeq\left(v_{\mathrm{h}} / \sqrt{2 \pi P r}\right)\left\{\ln \sqrt{P r u_{\mathrm{c}} / u_{\mathrm{v}}}\right\}^{1 / 2}$

For reasonably large $P r$, the non-dimensional velocity $U_{0}=u_{0} / v_{\mathrm{h}}$ is still considered of order unity and approximated, with high accuracy, by the formula proposed by Vázquez et al. [14], $U_{\mathrm{a}}=(0.38+0.67 \sqrt{\ln P r}) / \sqrt{2 \pi P r}$, shown in Fig. 5 with circles.

For the free convection flow around heated spheres the structure of the thermal plume and of the viscous region around the sphere will correspond to that of a point source of heat, if the sphere radius is small compared with $l_{\mathrm{h}}^{\prime}$. This is so in the distinguished limiting case, when $a / l_{\mathrm{h}}^{\prime}$ or, equivalently, when the Peclet number $\sigma=u_{0} a / \alpha$, which is $O(\sqrt{R a})$, is of order unity. In this case the flow field in the heated region around the sphere, of size of order $a$, can be described by the small Reynolds number flow field due to the velocity $u_{0}$ induced at the point source by the buoyancy forces acting in the thermal plume, but also influenced by the buoyancy forces acting in the inner region. In this regime, $\sigma$ of order unity, which is not analysed in this paper, the Nusselt number rises above its heat conduction value 2 by an amount of order unity. It is easy to show that for values of $\sigma \ll 1, N u-2=\sigma$. For values of $\sigma \gg 1$ the heated region around the sphere shrinks to a thin boundary layer, where the buoyancy forces are balanced by the viscous forces. Then, the analysis of Acrivos [3] applies, leading to the relation $N u=0.98 R a^{1 / 4}$ between $N u$ and the Rayleigh number $R a=G r P r$.

\section{References}

[1] B. Gebhart, Y. Jaluria, R.L. Mahajan, B. Sammakia (see Chapter 5), in: Buoyancy-Induced Flows and 
Transport, Hemisphere Publishing Corporation, Washngton, DC, 1988, p. 1001 (see Chapter 5).

[2] H.J. Merk, J.A. Prins, Thermal convection in laminary boundary layers, Appl. Sci. Res. 4 (1953) 11-24 Series A.

[3] A. Acrivos, A theoretical analysis of laminar natural convection heat transfer to non-Newtonian fluids, AIChE Journal 6 (1960) 584-590.

[4] J.M. Potter, N. Riley, Free convection from a heated sphere at large Grashof number, Journal of Fluid Mechanics 100 (1980) 769-783.

[5] S.N. Brown, C.J. Simpson, Collision phenomena in free-convective flow over a sphere, Journal of Fluid Mechanics 124 (1982) 123-127.

[6] J.J. Mahony, Heat transfer at small Grashof numbers, Proceeding of the Royal Society of London A238 (1957) 412-423.

[7] F. Fendell, Laminar natural convection about an isothermally heated sphere at small Grashof number, Journal of Fluid Mechanics 34 (1968) 163-176.

[8] C.A. Hieber, B. Gebhart, Mixed convection from a sphere at small Reynolds and Grashof numbers, Journal of Fluid Mechanics 38 (1969) 137-159.

[9] S. Kaplun, P.A. Lagerstrom, Asymptotic expansions of Navier-Stokes solutions for small Reynolds numbers, J. Math. Mech. 6 (1957) 585-593.

[10] A. Liñán, V.N. Kurdyumov, Laminar free convection induced by a line heat source, and heat transfer from wires at small Grashof numbers, Journal of Fluid Mechanics 362 (1998) 199-227.

[11] YaB Zeldovich, The Asymptotic laws of freely-ascending convective flows, Zhurnal eksperimentalnoi i teoreticheskoi fiziki 7 (1937) 1463-1465 [see also Selected Works of Yakov Borisovich Zeldovich, Princeton University Press, 1992].
[12] C.S. Yih, Free convection due to boundary sources, in: Fluid Models in Geophysics (Proceedings of the First Symposium on the Use of Models in Geophysics), United States Goverment Printing Office, Washington DC, 1956, pp. 117-133.

[13] T. Fujii, Theory of the steady laminar natural convection above a horizontal line heat source and a point heat source, International Journal of Heat and Mass Transfer 6 (1963) 597-606

[14] P.A. Vázquez, A.T. Pérez, A. Castellanos, Thermal and electrohydrodynamic plumes. A comparative study, Physics of Fluids 8 (1996) 2091-2096.

[15] T.G. Thomas, H.S. Takhar, Second-order effects in an axisymmetric plume, Quarterly Journal of Mechanics and Applied Mathematics 41 (1988) 1-16.

[16] D.S. Riley, D.G. Drake, Mixed convection in an axisymmetric buoyant plume, Quarterly Journal of Mechanics and Applied Mathematics 36 (1983) 43-54.

[17] F. Geoola, A.R.H. Cornish, Numerical solution of steady-state free convective heat transfer from a solid sphere, International Journal of Heat and Mass Transfer 24 (1981) 1369-1379.

[18] H. Jia, G. Gogos, Laminar natural convective heat transfer from isothermal spheres, International Journal of Heat and Mass Transfer 39 (1996) 16031615.

[19] F. Geoola, A.R.H. Cornish, Numerical simulation of free convective heat transfer from a sphere, International Journal of Heat and Mass Transfer 25 (1982) $1677-1687$.

[20] N. Riley, The heat transfer from a sphere in free convective flow, Computers \& Fluids 14 (1986) 225237.

[21] W. Schneider, Flow induced by jets and plumes, Journal of Fluid Mechanics 108 (1981) 55-65. 